\title{
ON EXCEEDANCE TIMES FOR SOME PROCESSES WITH DEPENDENT INCREMENTS
}

\author{
SØREN ASMUSSEN, * Aarhus University \\ SERGEY FOSS, ${ }^{* *}$ Heriot-Watt University and Sobolev Institute of Mathematics
}

\begin{abstract}
Let $\left\{Z_{n}\right\}_{n \geq 0}$ be a random walk with a negative drift and independent and identically distributed increments with heavy-tailed distribution, and let $M=\sup _{n \geq 0} Z_{n}$ be its supremum. Asmussen and Klüppelberg (1996) considered the behavior of the random walk given that $M>x$ for large $x$, and obtained a limit theorem, as $x \rightarrow \infty$, for the distribution of the quadruple that includes the time $\tau=\tau(x)$ to exceed level $x$, position $Z_{\tau}$ at this time, position $Z_{\tau-1}$ at the prior time, and the trajectory up to it (similar results were obtained for the Cramér-Lundberg insurance risk process). We obtain here several extensions of this result to various regenerative-type models and, in particular, to the case of a random walk with dependent increments. Particular attention is given to describing the limiting conditional behavior of $\tau$. The class of models includes Markov-modulated models as particular cases. We also study fluid models, the Björk-Grandell risk process, give examples where the order of $\tau$ is genuinely different from the random walk case, and discuss which growth rates are possible. Our proofs are purely probabilistic and are based on results and ideas from Asmussen, Schmidli and Schmidt (1999), Foss and Zachary (2002), and Foss, Konstantopoulos and Zachary (2007).
\end{abstract}

Keywords: Björk-Grandell model; Breiman's theorem; conditioned limit theorem; Markov modulation; mean excess function; random walk; regenerative process; regular variation; ruin time; subexponential distribution

2010 Mathematics Subject Classification: Primary 60K15; 60F10

Secondary 60E99; 60K25

\section{Introduction}

Let $Z=\{Z(t)\}_{t \geq 0}$ be a stochastic process with increments having a regenerative structure (see [2]): there exist random times $T_{0}=0, T_{1}, T_{2}, \ldots$ splitting $Z$ into independent and identically distributed (i.i.d.) cycles

$$
\{Z(t)-Z(0)\}_{0 \leq t<R_{1}}=\left\{Z\left(t+T_{0}\right)-Z\left(T_{0}\right)\right\}_{0 \leq t<R_{1}},\left\{Z\left(t+T_{k}\right)-Z\left(T_{k}\right)\right\}_{0 \leq t<R_{k+1}}, \ldots
$$

with lengths $R_{0}=T_{0}=0, R_{1}=T_{1}-T_{0}, R_{2}=T_{2}-T_{1}, \ldots$ (traditionally as in [2], one allows the first cycle to have a different distribution; we will not do this since our results are easily adapted to this setting). We will also assume that $Z(0)=0$. A main example we have in mind is the claims surplus process of an insurance company (accumulated claims minus premiums;

Received 1 June 2012; revision received 18 March 2013. Accepted by Onno Boxma, Coordinating Editor.

* Postal address: Department of Mathematics, Aarhus University, Ny Munkegade, DK-8000 Aarhus C, Denmark.

Email address: asmus@imf.au.dk

** Postal address: School of Mathematical and Computer Sciences, Heriot-Watt University, Edinburgh EH14 4AS,

UK. Email address: s.foss@hw.ac.uk

This author thanks the Kazakhstan Ministry of Education and Science (grant 1778/GF) for partial support. 
cf. [3]). In this setting, $\tau=\tau(x)=\inf \{t: Z(t)>x\}$ is the ruin time with initial surplus $x$, $M=\sup _{t \geq 0} Z(t)$ is the maximal claims surplus, and

$$
\mathbb{P}(\tau<\infty)=\mathbb{P}(M>x)
$$

is the ruin probability, but $\tau$ and $M$ are also of interest in many other contexts. For example, $M$ could be the stationary waiting time in a single-server queue with i.i.d. service times whose input process is modulated by a Markov chain (say, this is an output process from another stationary single-server queue; see, e.g. [9]).

Under suitable assumptions, the supremum

$$
\sup _{0 \leq t \leq R_{k+1}}\left(Z\left(t+T_{k}\right)-Z\left(T_{k}\right)\right),
$$

over a typical regenerative cycle of the process increments, has a heavy-tailed distribution, say $F$, on $[0, \infty)$ with mean $m_{F}<\infty$ whose integrated tail distribution

$$
\overline{F^{\mathrm{I}}}(x)=\min \left(1, \int_{x}^{\infty} \bar{F}(y) \mathrm{d} y\right)
$$

is subexponential. It has been proved in a variety of settings that

$$
\mathbb{P}(\tau<\infty)=\mathbb{P}(M>x) \sim b \overline{F^{\mathrm{I}}}(x) \quad \text { as } x \rightarrow \infty,
$$

where $b>0$ is a constant, thereby extending a classical result for random walks and the Cramér-Lundberg process due to (in alphabetical order) Borovkov, Cohen, Embrechts, Pakes, Veraverbeke, and von Bahr. In particular, Asmussen et al. [8] proved the following theorem (for background on subexponential distributions, see, e.g. [3, Section X.1], [18], or [21]).

Theorem 1.1. In the regenerative setting, let

$$
\xi_{k}=Z\left(T_{k+1}\right)-Z\left(T_{k}\right), \quad \xi_{k}^{*}=\sup _{T_{k} \leq t<T_{k+1}} Z(t)-Z\left(T_{k}\right)
$$

Assume that

$$
\mathbb{P}\left(\xi_{1}>x\right) \sim \mathbb{P}\left(\xi_{1}^{*}>x\right) \sim \bar{F}(x) \text { as } x \rightarrow \infty
$$

for some distribution $F$ such that $\overline{F^{\mathrm{I}}}$ is a subexponential tail, and that $-a=\mathbb{E} \xi_{1}<0$. Then

$$
\mathbb{P}(M>x) \sim \frac{1}{a} \overline{F^{\mathrm{I}}}(x) \text { as } x \rightarrow \infty .
$$

As demonstrated by the examples in [8] (and later papers, of which Asmussen and Biard [4] is a recent instance), this result covers a large number of examples. Foss and Zachary [19] gave a similar result in the case of a modulated random walk.

The purpose of the present paper is to supplement Theorem 1.1 and the corresponding result from [19] with a description of the asymptotic behavior of $\tau$ given $M>x$, but in a more general setting that covers both scenarios (of regenerative structure and of modulation). Results of this type were first given in Asmussen and Klüppelberg [5], where it was assumed that $Z$ is either the classical Cramér-Lundberg risk process, a Lévy process, or a discrete-time random walk $Z_{n}=\xi_{1}+\cdots+\xi_{n}$ with the $\xi_{k}$ i.i.d. and having common distribution $F$ and mean $-a<0$. Note that there is a discrete-time random walk imbedded in the regenerative setting: consider the process $Z$ at times $T_{n}$. 
In the random walk setting, the basic assumption of [5] is that there exists a function $e(x) \uparrow \infty$ such that, for any $t>0$,

$$
\lim _{x \rightarrow \infty} \frac{\overline{F^{\mathrm{I}}}(x+t e(x))}{\overline{F^{\mathrm{I}}}(x)}=\bar{G}(t)
$$

for some distribution $G$. We assume in addition that the function $e(x)$ is what could be called weakly self-neglecting, i.e.

$$
\limsup _{x \rightarrow \infty} \frac{e(x+e(x))}{e(x)}<\infty .
$$

Both assumptions (1.2) and (1.3) hold in the standard examples of subexponential distributions; see, e.g. [10] and [22] for further details. In the regularly varying case $\bar{F}(x)=L(x) / x^{\alpha}$, a natural scaling is $e(x)=x$; then (1.3) is automatic and $G$ is Pareto with $\bar{G}(t)=(1+t)^{-\alpha}$. For other subexponential distributions such as the lognormal and the heavy-tailed Weibull, we may take $e(x)=\bar{F}^{\mathrm{I}}(x) / \bar{F}(x)$ and then $G$ is standard exponential. Let $W$ be a random variable (RV) with distribution $G$. Then, with $\tau^{\mathrm{rw}}(x)=\inf \left\{n: Z_{n}>x\right\}$, the following theorem is shown in [5] (for later contributions in the same direction, see [6] and [23]).

Theorem 1.2. In the random walk setting suppose that $F^{\mathrm{I}}$ is a subexponential distribution and that (1.2) holds. Then, as $x \rightarrow \infty$, the conditional distribution of $\tau^{\mathrm{rw}}(x) / e(x)$ given $M>x$ converges to the distribution of $W / a$.

Our first main result is the following extension. For a stochastic process with the regenerative structure introduced above, for cycle $i$, let

$$
t_{i}=t_{i}(x)=\inf \left\{t \leq R_{i}: Z\left(t+T_{i-1}\right)-Z\left(T_{i-1}\right)>x\right\}
$$

if $\xi_{i}^{*}>x$, and $t_{i}=R_{i}$ otherwise.

Theorem 1.3. In the regenerative setting, in addition to assuming that the conditions of Theorem 1.1 and (1.2)-(1.3) hold, suppose that, for any $y>0$,

$$
\mathbb{P}\left(t_{1}>\text { ye }(x) \mid \xi_{1}>x\right)=\mathrm{o}(1) \text { as } x \rightarrow \infty .
$$

Then the conditional distribution of $\tau / e(x)$ given $M>x$ converges to the distribution of $\mu W / a$, where $\mu=\mathbb{E} R$.

The intuition behind Theorem 1.3 is as follows. In [5] a number of supplementary results are given that support the folklore that exceedance of level $x$ occurs as a result of one big $\xi_{k}$ and that all the other $\xi_{k}$ are 'typical'. In the regenerative setting, it was shown in [8] that the events $\tau<\infty$ and $\tau^{\mathrm{rw}}<\infty$ (where the random walk is the process observed at times $T_{n}$ ) are essentially equivalent, and that exceedance asymptotically occurs in cycle $\tau^{\mathrm{rw}}(x)$. Thus, we expect, by the law of large numbers, by the 'typical' behavior before $\tau^{\mathrm{rw}}$, and by (1.4) (which ensures that the length of the cycle in which ruin occurs can be neglected), that, conditionally on $M>x, \tau / \tau^{\mathrm{rw}} \rightarrow \mu$. Given this, Theorem 1.2 then yields the desired conclusion.

The technical problem is to make this intuition precise in this and in more general settings. A difficulty is that conditioning on $\tau$ introduces some (presumably) small dependence between cycles $1, \ldots, \tau^{\mathrm{rw}}-1$ as well as some bias in their distribution (expected to be small as well); this was realized in [4], with the consequence that some results there are heuristic. To overcome this difficulty, we present an approach for results of the type Theorem 1.2 which is novel and combines the ideas from [5] and a sample-path analysis developed in [9], [19], and [20]. The new 
approach is developed in Section 3 in the setting of random walks modulated by a regenerative process $Y$. For such a process, the asymptotics for $\mathbb{P}(M>x)$ are given in [20] (note that the setting allows $Y$ to be a Markov process with a general state space, whereas the setting in [8] can only handle the finite case). We supplement here with our second main result, Theorem 3.2, giving the conditional behavior of $\tau$. Compared to Theorem 1.3, it has the advantage that no conditions like (1.1) or (1.4) have to be verified, but it is also somewhat less general.

It is easy to construct examples where (1.4) fails as well as the conclusion of Theorem 1.3; see Section 7. The order of $\tau$ may remain $e(x)$ (then with a multiplier larger than $\mu W / a$ ) or be effectively larger. It is tempting to conjecture that any rate $\varphi(x)$ with $\varphi(x) / e(x) \rightarrow \infty$ may be attained. However, we will show that $1 / \bar{F}(x)$ is a critical upper bound.

\section{Preliminaries}

Definition 2.1. Let $F$ be a distribution function, and let $\bar{F}(x)=1-F(x)$ be its tail. Let $h(x)$ be a positive, nondecreasing function. We say that $F$ is $h$-insensitive if

$$
\bar{F}(x+h(x)) \sim \bar{F}(x) \text { as } x \rightarrow \infty .
$$

If (1.2) holds for $F$, we can take $h$ to be any function with $h(x)=\mathrm{o}(e(x))$. The reader is referred to [21, Chapter 2] for further information about the $h$-insensitivity property. The term $h$-flat is also used by some authors; see, e.g. [11].

Remark 2.1. Any subexponential distribution $F$ is long tailed, i.e. $\bar{F}(x+C) \sim \bar{F}(x)$ for any constant $C$. Therefore, by the diagonal argument, we can choose a positive function $h \uparrow \infty$ such that $F$ is also $h$-insensitive (clearly, the choice of $h$ depends on $F$ ). If $F$ is $h$-insensitive and $0 \leq g \leq h$, then $F$ is also $g$-insensitive.

Definition 2.2. We say that two families of events $A_{x}$ and $B_{x}$ of positive probabilities, indexed by $x>0$, are equivalent and write $A_{x} \sim B_{x}$ if $\mathbb{P}\left(A_{x} \Delta B_{x}\right)=\mathrm{o}\left(\mathbb{P}\left(A_{x}\right)\right)$ as $x \rightarrow \infty$, where $A \Delta B=A \backslash B \cup B \backslash A$ is the symmetric difference.

Note that if $A_{x} \sim B_{x}$ then $\mathbb{P}\left(A_{x}\right) \sim \mathbb{P}\left(B_{x}\right)$ also.

\section{Modulated random walk}

Consider a discrete-time regenerative process $Y=\left\{Y_{n}, n \geq 1\right\}$ such that, for each $n, Y_{n}$

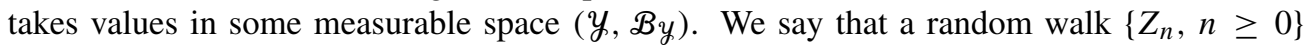
defined by $Z_{0}=0$ and $Z_{n}=\xi_{1}+\cdots+\xi_{n}$ for $n \geq 1$ is modulated by the process $Y$ if

(i) conditionally on $Y$, the $\mathrm{RVs} \xi_{n}, n \geq 1$, are independent;

(ii) for some family $\left\{F_{y}, y \in \mathcal{Y}\right\}$ of distribution functions such that, for each $x, F_{y}(x)$ is a measurable function of $y$, we have, for $n=1,2, \ldots$,

$$
\mathbb{P}\left(\xi_{n} \leq x \mid Y\right)=\mathbb{P}\left(\xi_{n} \leq x \mid Y_{n}\right)=F_{Y_{n}}(x) \text { almost surely (a.s.). }
$$

Let $M^{\mathrm{rw}}=\sup _{n \geq 0} Z_{n}$. Under conditions (C1)-(C4) below, $Z_{n} \rightarrow-\infty$ a.s. as $n \rightarrow \infty$, and so the random variable $M^{\mathrm{rw}}$ is finite a.s.

The regenerative epochs of the modulating process $Y$ are denoted by $0=T_{0}<T_{1}<\cdots$, with $R_{k}=T_{k}-T_{k-1}$. By definition, the cycles $\left(R_{k},\left(Y_{n}, 0<n \leq T_{k}-T_{k-1}\right)\right), k \geq 1$, are i.i.d. We assume that

$$
\mu=\mathbb{E} R_{1}<\infty
$$


Let

$$
\pi(B)=\frac{\mathbb{E} \sum_{1}^{R_{1}} \mathbf{1}\left(Y_{n} \in B\right)}{\mu}, \quad B \in \mathcal{B} y,
$$

be the stationary probability measure. We assume that each distribution $F_{y}, y \in \mathcal{y}$, has a finite mean

$$
a_{y}=\mathbb{E}\left[\xi_{n} \mid Y_{n}=y\right]=\int_{-\infty}^{\infty} x F_{y}(\mathrm{~d} x) \in(-\infty, \infty),
$$

and that the family of distributions

$$
\left\{F_{y}, y \in \mathcal{Y}\right\} \text { is uniformly integrable. }
$$

Furthermore, we assume that this family of distributions satisfies the following additional assumptions with respect to some reference distribution $F$ with finite mean and some measurable function $c: y \rightarrow[0,1]:$

(C1) $\overline{F_{y}}(x) \leq \bar{F}(x)$ for all $x \in \mathbb{R}$ and all $y \in \mathcal{y}$

(C2) $\overline{F_{y}}(x) \sim c(y) \bar{F}(x)$ as $x \rightarrow \infty$ for all $y \in y$;

(C3) $\kappa=\sup _{y \in y} a_{y}$ is finite and $a=-\int_{y} a_{y} \pi(\mathrm{d} y)$ is finite and strictly positive;

(C4) for some nonnegative $b>\kappa$,

$$
\mathbb{P}\left(b R_{1}>n\right)=\mathrm{o}(\bar{F}(n)) \quad \text { as } n \rightarrow \infty .
$$

Note that condition (C4) is redundant if $\kappa<0$-then we can take $b=0$.

The following result is known (see Theorem 2.2 of [20] for a slightly more general version and also for a discussion on the importance of conditions; see also Proposition 3.2 of [23]).

Theorem 3.1. Suppose that conditions (3.1)-(3.4) and (C1)-(C4) hold, and that the distribution $F^{\mathrm{I}}$ is subexponential. Then $Z_{n} / n \rightarrow-a$ a.s. as $n \rightarrow \infty$; in particular, $M^{\mathrm{rw}}$ is an almost-sure finite RV. Furthermore,

$$
\lim _{x \rightarrow \infty} \frac{\mathbb{P}\left(M^{\mathrm{rw}}>x\right)}{\overline{F^{\mathrm{I}}}(x)}=\frac{C}{a},
$$

where $C=\int_{y} c(y) \pi(\mathrm{d} y) \in[0,1]$.

The main idea in the proof of Theorem 3.1 is that the supremum of the modulated random walk, $M^{\mathrm{rw}}$, may be closely approximated by a sum of two independent RVs, where one has a light-tailed distribution and the other is the supremum of an ordinary random walk with i.i.d. heavy-tailed increments with integrated tail distribution proportional to $\bar{F}$.

We also note that, by the strong law of large numbers (SLLN) and the diagonal argument, we can choose a sequence $\varepsilon_{n} \downarrow 0$ such that

$$
\mathbb{P}\left(\left|Z_{m}+m a\right| \leq m \varepsilon_{m} \text { for all } m \geq n\right) \rightarrow 1 \quad \text { as } n \rightarrow \infty .
$$

Then, for any function $h(t) \uparrow \infty$ as $x \rightarrow \infty$,

$$
\mathbb{P}\left(\left|Z_{m}+m a\right| \leq m \varepsilon_{m}+h(x) \text { for all } m\right) \rightarrow 1 \text { as } x \rightarrow \infty .
$$

Based on Theorem 3.1 and (3.5), we obtain the following auxiliary result (see, e.g. Corollary 5 of [19] for an analogous statement in the case of an ordinary random walk). 
Proposition 3.1. Assume that the conditions of Theorem 3.1 hold and that $C>0$. Let the function $h(x) \uparrow \infty, h(x)=\mathrm{o}(x)$, be such that $F^{\mathrm{I}}$ is $h$-insensitive, and introduce the events

$$
\begin{gathered}
K_{n, x}=\bigcap_{m \leq n-1}\left\{\left|Z_{m}+m a\right| \leq m \varepsilon_{m}+h(x)\right\}, \\
A_{n, x}=\left\{\xi_{n}>x+n a\right\}, \quad A_{n, x}^{\varepsilon, h}=\left\{\xi_{n}>x+n a+n \varepsilon_{n}+h(x)\right\} .
\end{gathered}
$$

Then the following equivalences hold:

$$
\begin{aligned}
\left\{M^{\mathrm{rw}}>x\right\} & \sim \bigcup_{n \geq 1}\left\{M^{\mathrm{rw}}>x\right\} \cap A_{n, x} \cap K_{n, x} \\
& \sim \bigcup_{n \geq 1}\left\{M^{\mathrm{rw}}>x\right\} \cap A_{n, x}^{\varepsilon, h} \cap K_{n, x} \\
& \sim \bigcup_{n \geq 1}\left\{M^{\mathrm{rw}}>x\right\} \cap A_{n, x} \\
& \sim \bigcup_{n \geq 1}\left\{M^{\mathrm{rw}}>x\right\} \cap A_{n, x}^{\varepsilon, h} \\
& \sim \bigcup_{n \geq 1} A_{n, x} \\
& \sim \bigcup_{n \geq 1} A_{n, x}^{\varepsilon, h} ;
\end{aligned}
$$

therefore,

$$
\mathbb{P}\left(M^{\mathrm{rw}}>x\right) \sim \sum_{n \geq 1} \mathbb{P}\left(A_{n, x}^{\varepsilon, h} \cap K_{n, x}\right) \sim \sum_{n \geq 1} \mathbb{P}\left(A_{n, x}^{\varepsilon, h}\right) \sim \sum_{n \geq 1} \mathbb{P}\left(A_{n, x}\right) \sim \frac{C}{a} \overline{F^{I}}(x) .
$$

Finally, there exists a function $N=N(x) \rightarrow \infty$ such that $\bar{F}^{\mathrm{I}}(x+a N) \sim \bar{F}^{\mathrm{I}}(x)$ and equivalences (3.6) continue to hold if we replace $n \geq 1$ by $n \geq N$.

Proof. We can easily verify that

$$
\bigcup_{n \geq 1} K_{n, x} \cap A_{n, x}^{\varepsilon, h} \subseteq\left\{M^{\mathrm{rw}}>x\right\} .
$$

The events $K_{n, x} \cap A_{n, x}^{\varepsilon, h}$ are disjoint and $\sum_{n \geq 1} \mathbb{P}\left(A_{n, x}^{\varepsilon, h} \backslash A_{n, x}\right)=o\left(\overline{F^{\mathrm{I}}}(x)\right)$; hence, by (3.5),

$$
\mathbb{P}\left(\bigcup_{n \geq 1} K_{n, x} \cap A_{n, x}^{\varepsilon, h}\right)=\sum_{n \geq 1} \mathbb{P}\left(K_{n, x} \cap A_{n, x}^{\varepsilon, h}\right) \sim \sum_{n \geq 1} \mathbb{P}\left(K_{n, x} \cap A_{n, x}\right) \sim \sum_{n \geq 1} \mathbb{P}\left(A_{n, x}\right) .
$$

Since we have $\mathbb{P}\left(M^{\mathrm{rw}}>x\right) \sim(C / a) \overline{F^{\mathrm{I}}}(x)$ by Theorem 3.1, and since, by direct computations, $\sum_{n \geq 1} \mathbb{P}\left(A_{n, x}\right) \sim(C / a) \overline{F^{\mathrm{I}}}(x)$, equivalences (3.7) follow. The last fact follows directly from Remark 2.1 and equivalences (3.6) and (3.7). This completes the proof.

A special case of a modulated random walk is an ordinary random walk with i.i.d. increments. Consider an auxiliary i.i.d. sequence $\left\{\xi_{n}^{\sharp}\right\}$ with distribution $F$, and introduce the events

$$
A_{n, x}^{\sharp}=\left\{\xi_{n}^{\sharp}>x+n a\right\} \quad \text { and } \quad D_{x}^{\sharp}=\bigcup_{n \geq 1} A_{n, x}^{\sharp} .
$$


Assume that there exists a function $e(x) \uparrow \infty$ such that, for any $t>0$, there exists a limit

$$
\lim _{x \rightarrow \infty} \frac{\mathbb{P}\left(D_{x+t e(x)}^{\sharp}\right)}{\mathbb{P}\left(D_{x}^{\sharp}\right)}=\bar{G}(t)
$$

with $\lim _{t \rightarrow \infty} \bar{G}(t)=0$. Note that condition (3.8) is nothing other than condition (1.2) since $\mathbb{P}\left(D_{x}^{\sharp}\right) \sim \sum_{n \geq 1} \mathbb{P}\left(A_{n, x}^{\sharp}\right) \sim(1 / a) \bar{F}^{\mathrm{I}}(x)$.

On the event $D_{x}^{\sharp}$, introduce the random variable

$$
\tau^{\sharp} \equiv \tau^{\sharp}(x)=\min \left\{n \geq 1: \mathbf{1}\left(A_{n, x}^{\sharp}=1\right)\right\} .
$$

Then the following result holds.

Lemma 3.1. Assume that the distribution $F^{\mathrm{I}}$ is subexponential and that (1.2) holds. Then the conditional distribution of $\tau^{\sharp} / e(x)$ given $D_{x}^{\sharp}$ converges to the distribution $G$ (say of the $R V W$ ).

Indeed,

$$
\begin{aligned}
\mathbb{P}\left(\frac{a \tau^{\sharp}}{e(x)}>t \mid \tau^{\sharp}<\infty\right) & =\mathbb{P}\left(\tau^{\sharp}>\frac{t}{a} e(x) \mid \tau^{\sharp}<\infty\right) \\
& \sim \frac{\sum_{n>(t / a) e(x)} \mathbb{P}\left(\xi_{n}^{\sharp}>x+n a\right)}{\mathbb{P}\left(D_{x}^{\sharp}\right)} \\
& \sim \frac{\mathbb{P}\left(D_{x+t e(x)}^{\sharp}\right)}{\mathbb{P}\left(D_{x}^{\sharp}\right)} \\
& \rightarrow \bar{G}(t) .
\end{aligned}
$$

We now return to the modulated random walk. On the event $\left\{M^{\mathrm{rw}}>x\right\}$, we similarly introduce the RV

$$
\tau^{\mathrm{rw}}=\tau^{\mathrm{rw}}(x)=\min \left\{n \geq 1: Z_{n}>x\right\} .
$$

Recall from Proposition 3.1 that

$$
\left\{M^{\mathrm{rw}}>x\right\} \sim D_{x}=\bigcup_{n \geq 1} A_{n, x} .
$$

Then, by Lemma 3.1, we obtain the following result.

Lemma 3.2. Under the assumptions of Theorem 3.1 with $C>0$ and (1.2), the conditional distribution of a $\tau^{\mathrm{rw}} / e(x)$, conditioned on $\left\{M^{\mathrm{rw}}>x\right\}$, converges to the distribution $G$.

Indeed, the equivalence

$$
\mathbb{P}\left(a \tau^{\sharp}>t e(x) \mid \tau^{\sharp}<\infty\right) \sim \mathbb{P}\left(a \tau^{\mathrm{rw}}>t e(x) \mid \tau^{\mathrm{rw}}<\infty\right)
$$

holds since we may represent conditional probabilities as ratios of probabilities where both numerators and both denominators are pairwise asymptotically proportional, with the same coefficient $C$.

Furthermore, by Proposition 3.1 and Lemma 3.2, we deduce the following result.

Theorem 3.2. Assume that (1.2) holds. Then, under the conditions of Theorem 3.1 and the assumption that $C>0$, the distribution of

$$
\left(\frac{a \tau^{\mathrm{rw}}}{e(x)}, \frac{Z_{\tau^{\mathrm{rw}}-1}}{e(x)}, \max _{0 \leq m \leq \tau^{\mathrm{rw}}-1} \frac{\left|Z_{m}+m a\right|}{\tau^{\mathrm{rw}}}, \frac{Z_{\tau^{\mathrm{rw}}}-x}{e(x)}\right),
$$


conditioned on $\left\{M^{\mathrm{rw}}>x\right\}$, converges to the distribution of $\left(W,-W, 0, W^{\prime}\right)$, where $W$ and $W^{\prime}$ have the same distribution $G$ and, for any positive $u$ and $v$,

$$
\mathbb{P}\left(W>u, W^{\prime}>v\right)=\mathbb{P}(W>u+v) .
$$

This result is a complete analogue of Theorem 1.1 of Asmussen and Klüppelberg [5] which was obtained in the case of an ordinary random walk.

Proof of Theorem 3.2. We have already proved the convergence of the first component in (3.9). From that and (3.5), we conclude that

$$
\mathbb{P}\left(\left|Z_{m}+m a\right| \leq m \varepsilon_{m}+h(x) \text { for all } m<\tau^{\mathrm{rw}} \mid \tau^{\mathrm{rw}}<\infty\right) \rightarrow 1 \text { as } x \rightarrow \infty .
$$

Then the convergence of the second and third components in (3.9) follows if we take $h(x) \rightarrow \infty$ such that $h(x)=\mathrm{o}(e(x))$.

It remains to show the convergence of the last component in (3.9). Since

$$
\left\{Z_{\tau^{\mathrm{rw}}}-x>v e(x)\right\} \sim \bigcup_{n \geq 1}\left\{\xi_{n}-x-n a>v e(x)\right\},
$$

we obtain

$$
\begin{aligned}
\mathbb{P}\left(Z_{\tau^{\mathrm{rw}}}-x>v e(x)\right) & \sim \mathbb{P}\left(\bigcup_{n \geq 1}\left\{\xi_{n}-x-n a>v e(x)\right\}\right) \\
& \sim \sum_{n \geq 1} \mathbb{P}\left(\xi_{n}-x-n a>v e(x)\right) \\
& =\sum_{n \geq 1} \mathbb{P}\left(\xi_{n}>x+v e(x)+n a\right) \\
& \sim \mathbb{P}\left(D_{x+v e(x)}\right)
\end{aligned}
$$

(here we assume that $Z_{\tau^{\mathrm{rw}}}=-\infty$ if $\tau^{\mathrm{rw}}=\infty$ ). Similarly, equality (3.10) follows since

$$
\begin{aligned}
\left\{a \tau^{\mathrm{rw}}>\operatorname{ue}(x), Z_{\tau^{\mathrm{rw}}}-x>v e(x)\right\} & \sim \bigcup_{n>u e(x)}\left\{\xi_{n}>x+n a, \xi_{n}-n a-x>v e(x)\right\} \\
& =\bigcup_{n>u e(x)}\left\{\xi_{n}>x+n a+v e(x)\right\} .
\end{aligned}
$$

Then

$$
\begin{aligned}
\mathbb{P}\left(a \tau^{\mathrm{rw}}>u e(x), Z_{\tau^{\mathrm{rw}}}-x>v e(x)\right) & \sim \sum_{n>u e(x)} \mathbb{P}\left(\xi_{n}>x+n a+v e(x)\right) \\
& \sim C \sum_{n>u e(x)} \mathbb{P}\left(\xi_{n}^{\sharp}>x+n a+v e(x)\right) \\
& =C \sum_{n \geq 1} \mathbb{P}\left(\xi_{n}^{\sharp}>x+n a+(v+u) e(x)\right) \\
& \sim C \mathbb{P}\left(D_{x+(u+v) e(x)}^{\sharp}\right) \\
& \sim \mathbb{P}\left(D_{x+(u+v) e(x)}\right),
\end{aligned}
$$

completing the proof. 


\section{Continuous-time modulated regenerative processes}

We now consider the continuous-time process $Z(t)$ introduced in Section 1 and assume that, more generally, it is a regenerative process which is modulated by a discrete-time regenerative process $Y$. This means that (compare with the previous section!)

(i) conditionally on $Y$, the random elements $V_{k+1}=\left\{Z(t)-Z\left(T_{k}\right), 0 \leq t \leq R_{k+1}\right\}$ are independent;

(ii) for any $n$,

$$
\mathbb{P}\left(V_{n} \in \cdot \mid Y\right)=\mathbb{P}\left(V_{n} \in \cdot \mid Y_{n}\right) \text { a.s. }
$$

Furthermore, let, as in Theorem 1.1,

$$
\xi_{k}=Z\left(T_{k+1}\right)-Z\left(T_{k}\right), \quad \xi_{k}^{*}=\sup _{T_{k} \leq t<T_{k+1}} Z(t)-Z\left(T_{k}\right),
$$

and assume that the conditions of Theorem 3.1 and (1.2) hold. Then the statements of Theorems 3.1 and 3.2 also hold.

Note that $M \equiv \sup _{t \geq 0} Z(t)$ may also be represented as $M=\sup _{n \geq 0}\left(\xi_{1}+\cdots+\xi_{n}+\xi_{n+1}^{*}\right)$. Then we have the following result.

Theorem 4.1. Assume that the conditions of Theorem 3.1 and (1.2) hold, and that $C>0$ in Theorem 3.1. Assume further that, for all $y \in \mathcal{Y}$,

$$
\mathbb{P}\left(\xi_{n}^{*}>x \mid Y=y\right) \sim \bar{F}_{y}(x) \text { a.s. }
$$

and that

$$
\mathbb{P}\left(\xi_{n}^{*}>x\right) \leq c \bar{F}(x)
$$

for some $c \geq 1$ and all $x$. Then, as $x \rightarrow \infty$,

$$
\{M>x\} \sim\left\{M^{\mathrm{rw}}>x\right\} \sim \bigcap_{n \geq 1} K_{n, x} \cap A_{n, x}
$$

and, for $\hat{\tau}^{\mathrm{rw}} \equiv \hat{\tau}^{\mathrm{rw}}(x)=\min \left\{n \geq 1: Z_{n-1}+\xi_{n}^{*}>x\right\}$,

$$
\mathbb{P}\left(\tau^{\mathrm{rw}}=\hat{\tau}^{\mathrm{rw}} \mid M>x\right) \rightarrow 1 \text { as } x \rightarrow \infty
$$

and

$$
\mathbb{P}\left(\tau^{\mathrm{rw}}=\hat{\tau}^{\mathrm{rw}} \mid M^{\mathrm{rw}}>x\right) \rightarrow 1 \text { as } x \rightarrow \infty .
$$

Therefore, the statement of Theorem 3.2 continues to hold if in (3.9) we replace $\tau^{\mathrm{rw}}$ by $\hat{\tau}^{\mathrm{rw}}$ and then $Z_{\tau^{\mathrm{rw}}}$ by $Z_{\hat{\tau}^{\mathrm{rw}}-1}+\xi_{\hat{\tau}^{\mathrm{ww}}}^{*}$.

The proof of Theorem 4.1 follows from minor, routine modifications of the calculations given in the previous section.

\section{Proof of Theorem 1.3}

Now we assume that the process $Z(t)$ is regenerative. This means that $Y$ is a constant and, as a corollary, conditions (3.1)-(3.4) and (C1)-(C4) are redundant. Also, the $\xi_{n}$ are i.i.d. in this case and, therefore, we may take $\tau^{\sharp}=\min \left\{n: \xi_{n}>x+n a\right\}$.

Let $\mathbb{P}^{(x)}$ denote the conditional probability given $\tau<\infty$, write $T_{n}=R_{1}+\cdots+R_{n}$, and recall the definition of $\hat{\tau}^{\mathrm{rw}}$ from Theorem 4.1. Note that, since the events $\tau<\infty$ and $\hat{\tau}^{\mathrm{rw}}<\infty$ coincide, and are equivalent to each of the events $\tau^{\mathrm{rw}}<\infty$ and $\tau^{\sharp}<\infty$ (see Lemma 3.2), we may use either of the four in conditioning arguments. 
The proof of Theorem 1.3 is a straightforward combination of Theorem 3.2, Theorem 4.1 and the following two lemmas. Both lemmas use the fact, implicit in [8] and also a consequence of (4.1) and (4.2), that

$$
\mathbb{P}^{(x)}\left(\tau \in\left[T_{\tau^{\mathrm{rw}}-1}, T_{\tau^{\mathrm{rw}}}\right)\right) \rightarrow 1 \quad \text { as } x \rightarrow \infty
$$

since, asymptotically,

$$
\left\{\tau \in\left[T_{\tau^{\mathrm{rw}}-1}, T_{\left.\tau^{\mathrm{rw}}\right)}\right) \subseteq\left\{\tau^{\mathrm{rw}}=\hat{\tau}^{\mathrm{rw}}\right\} .\right.
$$

Lemma 5.1. It holds that $T_{\tau^{\mathrm{rw}}-1} / e(x) \rightarrow \mu W / a$ in $\mathbb{P}^{(x)}$-distribution.

Proof. We use the representation

$$
\frac{T_{\tau^{\mathrm{rw}}-1}}{e(x)}=\frac{T_{\tau^{\mathrm{rw}}-1}}{\tau^{\mathrm{rw}}} \frac{\tau^{\mathrm{rw}}}{e(x)} .
$$

Choose $N=N(x) \rightarrow \infty$ from Proposition 3.1. The first fraction on the right-hand side of (5.1) converges to $\mu$ in $\mathbb{P}^{(x)}$ probability since, by the independence of $A_{n, x}$ and $T_{n-1}$ and by the SLLN,

$$
\begin{aligned}
\left\{\left|\frac{T_{\tau^{\mathrm{rw}}-1}}{\tau^{\mathrm{rw}}}-\mu\right| \leq \varepsilon, \tau^{\mathrm{rw}}<\infty\right\} & \sim \bigcup_{n \geq 1}\left\{\left|\frac{T_{n-1}}{n}-\mu\right| \leq \varepsilon\right\} \cap A_{n, x} \\
& \sim \bigcup_{n \geq N}\left\{\left|\frac{T_{n-1}}{n}-\mu\right| \leq \varepsilon\right\} \cap A_{n, x} \\
& \sim \bigcup_{n \geq N} A_{n, x} \\
& \sim \bigcup_{n \geq 1} A_{n, x} \\
& \sim\left\{\tau^{\mathrm{rw}}<\infty\right\} .
\end{aligned}
$$

Then the second fraction converges to $W / a$ by Theorem 3.2 , and the result follows.

Recall that $\tau=\sum_{1}^{\hat{\tau}^{\mathrm{rw}}-1} R_{i}+t_{\hat{\tau}^{\mathrm{rw}}}$.

Lemma 5.2. Under the conditions of Theorem 1.3, $t_{\hat{\tau}^{\mathrm{rw}}} / e(x) \rightarrow 0$ in $\mathbb{P}^{(x)}$-probability.

Proof. By Theorem 3.2 and Theorem 4.1, for any $\delta>0$, we can choose $K>0$ such that

$$
\mathbb{P}\left(\frac{\hat{\tau}^{\mathrm{rw}}}{e(x)}>K\right) \leq \frac{\delta}{2}
$$

for all large enough $x$. Then, for any $y>0$,

$$
\mathbb{P}^{(x)}\left(\frac{t_{\hat{\tau}^{\mathrm{rw}}}}{e(x)}>y\right) \leq(1+\mathrm{o}(1)) \frac{\sum_{n \leq K e(x)} \mathbb{P}\left(t_{n}>y e(x), \xi_{n}>x+n a\right)}{\bar{F}^{\mathrm{I}}(x) / a}+\delta .
$$

If (1.3) holds then $\lim \sup _{x \rightarrow \infty} e(x+k e(x)) / e(x)<\infty$ for any $k>0$. Therefore, the latter sum in the numerator is equivalent to

$$
\sum_{n \leq K e(x)} \mathbb{P}\left(t_{n}>y e(x+n a) \mid \xi_{n}>x+n a\right) \mathbb{P}\left(\xi_{n}>x+n a\right)=\mathrm{o}(1) \bar{F}^{\mathrm{I}}(x)
$$

since the $\left(t_{n}, \xi_{n}\right)$ are i.i.d. Then we complete the proof by first letting $x \rightarrow \infty$ and then $\delta \rightarrow 0$.

Combining this with the statements of Theorem 4.1 and Lemma 5.1 completes the proof of Theorem 1.3. 


\section{Examples}

Example 6.1. In the setting of Section 3, we assume that $Z(t)$ is a right-continuous, piecewise constant process with $Z(n)=Z_{n}$. We will show here that, under a natural extra assumption, Theorem 1.3 holds for this model as well. Note that, because of the result in [20], we need not verify the conditions of Theorem 1.1 (which may be messy); all that is needed is to establish (1.4).

Assume in addition that the distribution of the cycle length, $R$, has a lighter tail than $\bar{F}(x)$, in the following strong sense: there exists a constant $c>1$ such that

$$
\mathbb{P}(c R>x)=\mathrm{o}(\bar{F}(x)) \quad \text { as } x \rightarrow \infty .
$$

Let $\xi$ be the increment over the cycle, i.e. $\xi=\sum_{1}^{R} \xi_{i}$. For (1.4) to hold, it suffices to show that, for any $y>0$,

$$
\mathbb{P}(R>y x, \xi>x)=\mathrm{o}(\bar{F}(x)) \quad \text { as } x \rightarrow \infty,
$$

where $F$ is the reference distribution. For any fixed $x_{0}$ and $x \geq x_{0}$, as $x \rightarrow \infty$,

$$
\begin{aligned}
\mathbb{P}(R>y x, \xi>x) & =\mathbb{E}\left[\mathbb{P}\left(\xi>x \mid R, Y_{0}, \ldots, Y_{R}\right) \mathbf{1}(Y>y x)\right] \\
& \leq \sum_{k \geq x y} \overline{F^{k}}(x) \mathbb{P}(R=k) \quad(\text { by }(\mathrm{C} 2)) \\
& \leq \sum_{k \geq x_{0} y} \overline{F^{k}}(x) \mathbb{P}(R=k) \\
& \sim \bar{F}(x) \mathbb{E}\left[R ; R>x_{0} y\right],
\end{aligned}
$$

where, under assumption (6.1), the last equivalence follows from [17, Theorem 1]. By letting $x_{0} \rightarrow \infty$, we obtain (6.2).

Example 6.2. The Björk-Grandell model (see [13]) is a regenerative risk process such that, in addition to the cycle length $R$, the rate $\Lambda$ of claim arrivals within a cycle is random. All claims are i.i.d. with distribution $H$ and with mean $m$, and independent of $(R, \Lambda)$, with a constant rate 1 of premium inflow. The infinite-horizon ruin probabilities are discussed in [13] for the light-tailed case and in [8] for the heavy-tailed case. As noted in [8], heavy tails of $\xi$ may occur in at least three ways: (i) $F$ is heavy tailed; (ii) $\Lambda$ is heavy tailed; (iii) $R$ is heavy tailed for sufficiently large values of $\Lambda$. Under some (not necessarily minimal) assumptions, we will give arguments to identify the limiting conditional behavior of $\tau$.

For the following estimates, we may keep in mind

$$
\xi=X_{1}+\cdots+X_{M_{R \Lambda}}-R
$$

with $M$ an independent Poisson process at unit rate. For the tail asymptotics of $\xi$, the $-R$ term may often be neglected (see [4] for some preliminary discussion and [1] for a more complete picture). Also, with light-tailed claims we may frequently approximate $X_{1}+\cdots+X_{M_{R \Lambda}}$ by $m R \Lambda$; the relevant large deviations arguments are given in detail in [8] and will not be repeated here.

Consider case (i) with $R$ and $\Lambda$ both light tailed. Using (6.3) and an independence result from [4], it is standard that

$$
\mathbb{P}(\xi>x) \sim \mathbb{E}(R \Lambda) \bar{F}(x)
$$


By a classical inequality due to Kesten (see, e.g. [12, p. 429]), to each $\delta>0$ there is a $C_{\delta}<\infty$ such that $\mathbb{P}\left(X_{1}+\cdots+X_{n}>x\right) \leq C_{\delta} \mathrm{e}^{n \delta} \bar{F}(x)$ for all $n$. With

$$
p=\mathbb{P}\left(M_{R \Lambda}=1\right)>0, \quad q=p \mathbb{P}\left(X_{1}>x+R\right) \sim p \bar{F}(x),
$$

we obtain

$$
\begin{aligned}
\mathbb{E}\left[\mathrm{e}^{s R} \mid \xi>x\right] & =\frac{\mathbb{E}\left[\mathrm{e}^{s R} ; \xi>x\right]}{\mathbb{P}(\xi>x)} \\
& \leq \frac{1}{q} \mathbb{E}\left[\mathrm{e}^{s R} ; X_{1}+\cdots+X_{M_{R \Lambda}}>x\right] \\
& \leq \frac{1}{q} \mathbb{E}\left[\mathrm{e}^{s R} C_{\delta} \mathrm{e}^{\delta M_{R \Lambda}} \bar{F}(x)\right] \\
& \sim \frac{C_{\delta}}{p} \mathbb{E}\left[\mathrm{e}^{s R} \mathrm{e}^{R \Lambda\left(\mathrm{e}^{\delta}-1\right)}\right] .
\end{aligned}
$$

Taking $s$ and $\delta$ small enough, this expression is finite, and its independence of $x$ together with $e(x) \rightarrow \infty$ then gives (1.4) and the conclusion of Theorem 1.3.

Let us now consider case (ii) with $F$ light tailed and $(R, \Lambda)$ satisfying $\mathbb{P}(\Lambda>x) \sim x^{-\alpha}$ with $\alpha>1$ and $\mathbb{E} R^{\alpha^{\prime}}<\infty$ for some $\alpha^{\prime}>\alpha$. Then, by Breiman's theorem (see [14], [15], and [16] $), \mathbb{P}(m R \Lambda>x) \sim c x^{-\alpha}$, where $c=m^{\alpha} \mathbb{E} R^{\alpha}$. By a large deviations argument,

$$
\mathbb{P}\left(M_{R \Lambda}>x\right)=\mathbb{P}(R \Lambda>x)+\mathrm{O}\left(\mathrm{e}^{-\varepsilon_{1} x}\right)
$$

for some $\varepsilon_{1}>0$. A further large deviations argument, given in [7], then shows that

$$
\left\{X_{1}+\cdots+X_{M_{R \Lambda}}>x\right\} \Delta\{m R \Lambda>x\}=A(x),
$$

where $\mathbb{P} A(x)=\mathrm{O}\left(\mathrm{e}^{-\varepsilon x}\right)$ for some $\varepsilon>0$. In particular, $X_{1}+\cdots+X_{M_{R \Lambda}}$ has asymptotic tail $c x^{-\alpha}$. Hence, so has $\xi=X_{1}+\cdots+X_{M_{R \Lambda}}-R$ (see [4]; note that this is nontrivial due to dependence). Let $\alpha<\alpha^{\prime \prime}<\alpha^{\prime}$, and let $R^{*}$ be an RV with distribution

$$
\mathbb{P}\left(R^{*} \in \mathrm{d} t\right)=\frac{\mathbb{E}\left[R^{\alpha^{\prime \prime}} ; R \in \mathrm{d} t\right]}{\mathbb{E}\left(R^{\alpha^{\prime \prime}}\right)} .
$$

Then

$$
\begin{aligned}
\mathbb{E}\left[R^{\alpha^{\prime \prime}} \mid \xi>x\right] & \sim \frac{\mathbb{E}\left[R^{\alpha^{\prime \prime}} ; \xi>x\right]}{c x^{-\alpha}} \\
& \leq \frac{1}{c x^{-\alpha}} \mathbb{E}\left[R^{\alpha^{\prime \prime}} ; X_{1}+\cdots+X_{M_{R \Lambda}}>x\right] \\
& =\frac{1}{c x^{-\alpha}} \mathbb{E}\left[R^{\alpha^{\prime \prime}} ; m R \Lambda>x\right]+\mathrm{O}\left(\mathrm{e}^{-\varepsilon x}\right) \\
& =\frac{1}{c x^{-\alpha}} \mathbb{E} R^{\alpha^{\prime \prime}} \mathbb{P}\left(R^{*} \Lambda>\frac{x}{m}\right)+\mathrm{O}\left(\mathrm{e}^{-\varepsilon x}\right) .
\end{aligned}
$$

Another application of Breiman's theorem justified by the choice of $\alpha^{\prime \prime}$ shows that $R^{*} \Lambda$ has a distribution tail asymptotically proportional to $x^{-\alpha}$. Hence, $\mathbb{E}\left[R^{\alpha^{\prime \prime}} \mid \xi>x\right]$ stays bounded as $x \rightarrow \infty$, and arguing as above gives (1.4) and the conclusion of Theorem 1.3.

In contrast, the behavior in case (iii) is different; see Section 7.

Example 6.3. Let $Z$ be a two-stage fluid model, where a cycle $R$ is composed of two stages such that the first has deterministic length $a_{1}$ and the second a random length $R_{2}$ with a subexponential distribution $F$ with mean $a_{2}<a_{1}$. In stage $1, Z$ decreases deterministically at rate 1 and in 
stage $2, Z$ increases deterministically at rate 1 (thus, $-a=a_{2}-a_{1}<0$ ). Clearly, $\xi>x$ occurs if and only if $R_{2}>x+a_{1}$. Thus, $\mathbb{P}(\xi>x)=\bar{F}\left(x+a_{1}\right) \sim \bar{F}(x)$ and given $\xi>x, R$ is at least $x$. Since $e(x)=\mathrm{O}(x)$ in all examples, condition (1.4) cannot hold and, more precisely, given $\xi>x, R$ is of order $x+e(x)$. Therefore, $\tau$ is of order $e(x)$ in the regularly varying case, but with a multiplier larger than $\mu W$, and of order $x \gg e(x)$ for other subexponential distributions.

Note that this example shows that the regenerative setting is more flexible than the Markov additive setting: if we consider the discrete-time analogue, the increments in each Markov stage are bounded and there is thus no version of condition $(\mathrm{C} 2)$ with $F$ heavy tailed. On the other hand, conditions may be easier to verify in the Markov additive setting.

\section{Different growth rates}

The following result is straightforward given Lemma 5.1 and the proof of Theorem 1.3. For simplicity (to avoid distinction between $x$ and $e(x)$ ), we state it only for the regularly varying case where the RV $W$ in Theorem 1.2 is Pareto.

Corollary 7.1. Assume that $F$ in (1.1) is regularly varying and that instead of condition (1.4) we have

$$
\mathbb{P}\left(\frac{t_{1}}{e^{*}(x)}>y \mid \xi>x\right) \rightarrow \mathbb{P}\left(W^{*}>y\right) \text { for all } y
$$

for some function $e^{*}(x)$ with $\lim \inf e^{*}(x) / x>0$ and some $R V W^{*}$.

(i) If $e^{*}(x) \sim d x$ for some $d$ then

$$
\frac{\tau}{x} \rightarrow \frac{W \mu}{a}+d(1+W) W^{*} \text { in } \mathbb{P}^{(x)} \text {-distribution. }
$$

(ii) If $e^{*}(x) / x \rightarrow \infty$ then

$$
\frac{\tau}{e^{*}(x(1+W))} \rightarrow W^{*} \text { in } \mathbb{P}^{(x)} \text {-distribution }
$$

with $W$ and $W^{*}$ independent in both (i) and (ii), and $W$ independent of $\tau$ in (ii). In particular, if $e^{*}(x) \sim d x^{\beta}$ with $\beta>1$ then $\tau / x^{\beta} \rightarrow d(1+W)^{\beta} W^{*}$ in $\mathbb{P}^{(x)}$-distribution.

Proof. The asymptotic $\mathbb{P}^{(x)}$-distribution of $\tau$ is the same as the asymptotic distribution of $\sum_{1}^{\hat{\tau}^{\mathrm{rW}}-1} R_{i}+t_{\hat{\tau}^{\mathrm{rw}}}$. Here $\sum_{1}^{\hat{\tau}^{\mathrm{rw}}-1} R_{i} / x \rightarrow \mu W / a$ in $\mathbb{P}^{(x)}$-distribution (Lemma 5.1). More generally,

$$
\frac{1}{x}\left(\sum_{1}^{\hat{\tau}^{\mathrm{rw}}-1} R_{i}, \sum_{1}^{\hat{\tau}^{\mathrm{rw}}-1} \xi_{i}\right) \rightarrow\left(\frac{\mu W}{a}, W\right) .
$$

Given $W=w, \xi_{\hat{\tau}^{\mathrm{rw}}}$ will asymptotically have to exceed $x w+x$, implying that $t_{\widehat{\tau}^{\mathrm{rw}}} / e^{*}(x(w+$ 1)) $\rightarrow W^{*}$ and the conclusion of (i) since the limit $W^{*}$ does not depend of $w$. For (ii), just note that in this case $\sum_{1}^{\hat{\tau}^{\mathrm{rw}}-1} R_{i}$ may be neglected.

We now give an example of $e^{*}(x) \sim d x$ and thereafter some discussion of what may happen if $e^{*}(x) / x \rightarrow \infty$.

Example 7.1. We return to the Björk-Grandell model of Example 6.2(iii), i.e. $R$ is heavy tailed for sufficiently large values of $\Lambda$. Here we expect that given $\Lambda=\lambda$, the surplus process $\sum_{1}^{N(t)} U_{i}-t$ can be approximated by $\lambda m t-t$, and this is confirmed by the large deviations 
bounds in [8]. Therefore, the behavior should be like a fluid model with heavy-tailed on periods, so that the exceedance time of $x$ within a cycle should be of order $x$ and accordingly makes a genuine contribution to $\tau$.

We next verify this statement and make it more precise. Assume, as in [8], that the claims are light tailed and independent of $(R, \Lambda)$; that, for some $\lambda_{0}>1 / \mu$,

$$
\begin{array}{ll}
\mathbb{P}(R>t \mid \Lambda=\lambda)=\bar{F}(t), & \lambda>\lambda_{0}, \\
\mathbb{P}(R>t \mid \Lambda=\lambda) \leq \bar{G}(t), & \lambda \leq \lambda_{0},
\end{array}
$$

for some regularly varying $F$ with $\bar{F}(t)=L(t) / t^{\alpha}$ ( $L$ slowly varying) and some $G$ satisfying $\bar{G}(t)=\mathrm{o}(\bar{F}(t))$; and that the regularity condition

$$
\sup _{x \geq x_{0}} \frac{L(x / y)}{L(x)} \leq g(y)
$$

for all $y>0$, some $x_{0}>0$, and some function $g(y)$ with $\mathbb{E}\left[\Lambda^{\alpha} g(\Lambda)\right]<\infty$ holds.

It was then shown in [8] that the conditions of Theorem 1.1 are satisfied and that

$$
\psi(x) \sim c_{1} \bar{F}(x),
$$

where

$$
c_{1}=\frac{c}{(\alpha-1)[\mathbb{E} R-m \mathbb{E}(\Lambda R)]}, \quad c=\mathbb{E}\left[(\Lambda m-1)^{\alpha} ; \Lambda>\lambda_{0}\right] .
$$

This depends on the estimate

$$
\mathbb{P}(\xi>x) \sim c \bar{F}(x)
$$

As preparation for the study of the ruin time, we first recall the proof of (7.2). That the event $\xi>x$ occurs follows by large deviations arguments equivalent to $R(\Lambda m-1)>x$, and so

$$
\begin{aligned}
\mathbb{P}(\xi>x) & \sim \int_{\lambda_{0}}^{\infty} f_{\Lambda}(\lambda) \bar{F}\left(\frac{x}{\lambda m-1}\right) \mathrm{d} \lambda \\
& =\int_{\lambda_{0}}^{\infty} f_{\Lambda}(\lambda)(\lambda m-1)^{\alpha} \frac{L(x /(\lambda m-1))}{x^{\alpha}} \mathrm{d} \lambda \\
& \sim \int_{\lambda_{0}}^{\infty} f_{\Lambda}(\lambda)(\lambda m-1)^{\alpha} \frac{L(x)}{x^{\alpha}} \mathrm{d} \lambda \\
& =c \bar{F}(x),
\end{aligned}
$$

where the last ' $\sim$ ' follows by dominated convergence justified by (7.1). If $\xi>x, \tau \leq x t$ is to occur, we need in addition $x /(\lambda m-1) \leq x t$, and so, by the same dominated convergence argument,

$$
\begin{aligned}
\mathbb{P}(\xi>x, \tau \leq x t) & \sim \int_{\lambda_{0} \vee(1 / t+1) / m}^{\infty} f_{\Lambda}(\lambda) \bar{F}\left(\frac{x}{\lambda m-1}\right) \mathrm{d} \lambda \\
& \sim \int_{\lambda_{0} \vee(1 / t+1) / m}^{\infty} f_{\Lambda}(\lambda)(\lambda m-1)^{\alpha} \frac{L(x)}{x^{\alpha}} \mathrm{d} \lambda \\
& =c \bar{F}(x) W^{*}(t),
\end{aligned}
$$


where $W^{*}$ is the distribution with cumulative distribution function

$$
\mathbb{P}\left(W^{*} \leq t\right)= \begin{cases}\frac{1}{c} \int_{(1 / t+1) / m}^{\infty} f_{\Lambda}(\lambda)(\Lambda m-1)^{\alpha} \mathrm{d} \lambda, & t \leq 1 /\left(\lambda_{0} m-1\right), \\ 1, & t>1 /\left(\lambda_{0} m-1\right) .\end{cases}
$$

From Corollary 7.1 we therefore conclude that $\tau(x) / x \rightarrow W^{*}(1+W)$ in $\mathbb{P}^{(x)}$-distribution.

We now discuss when $\tau$ may grow at rates larger than $e(x)$ and how fast the rate may be. If $t_{x}=\mathbb{E}[R \mid \xi=x] \rightarrow \infty$ faster than $e(x)$, we expect $R$ given $\xi>x$ (and, hence, often $\tau$ ) to grow at a faster rate than $e(x)$. At first sight, we could conjecture that any rate is possible. This is, however, not possible because of the requirement that $\mathbb{E} R<\infty$. Suppose, for example, that $F$ is a discrete subexponential distribution with point probabilities $f_{x}=\mathbb{P}(\xi=x) \sim c_{1} / x^{\alpha+1}$. Assuming that $t_{x} \sim c_{2} x^{\beta}$, we obtain

$$
\infty>\mathbb{E} R=\sum_{0}^{\infty} t_{x} f_{x} \approx \sum_{0}^{\infty} c_{2} x^{\beta} \frac{c_{1}}{x^{\alpha+1}}
$$

implying that $\beta<\alpha$. The following result gives the more precise upper bound $c / \bar{F}(x)$ and is more satisfying by being in terms of the growth rate of $\tau$ rather than expected values.

Theorem 7.1. Let $F(x)=\mathbb{P}(\xi \leq x)$ be a discrete subexponential distribution with point probabilities $f_{0}, f_{1}, \ldots$, and let $\varphi$ be a function with $\varphi(x) / e(x) \rightarrow \infty$. Assume that $\mathbb{P}(R>$ $\varepsilon \varphi(x) \mid \xi>x) \geq \delta$ for some $\varepsilon, \delta>0$ and all large $x$. Then $\varphi(x) \leq c / \bar{F}(x)$ for some constant $c$.

Proof. Define $t(x)$ as above, and let

$$
k(x)=\mathbb{E}[R \mid \xi>x]=\frac{1}{\bar{F}(x)}\left(t_{x+1} f_{x+1}+t_{x+2} f_{x+2}+\cdots\right) .
$$

Multiplying by $\bar{F}(x)$ and subtracting the resulting equation with $x$ replaced by $x+1$, it follows that

$$
t_{x+1}=\frac{1}{f_{x+1}}(k(x) \bar{F}(x)-k(x+1) \bar{F}(x+1)) .
$$

This expression needs to be positive which gives $k(x+1) / k(x)<\bar{F}(x) / \bar{F}(x+1)$ and, multiplying from $x=1$ to $y-1$,

$$
k(y)<k(1) \bar{F}(1) \frac{1}{\bar{F}(y)} .
$$

However, clearly, $k(x)=\mathbb{E}[R \mid \xi>x] \geq \varepsilon \delta h(x)$, from which we conclude that

$$
\varphi(x) \leq \frac{k(x)}{\varepsilon \delta}<\frac{k(1) \bar{F}(1)}{\varepsilon \delta} \frac{1}{\bar{F}(x)},
$$

completing the proof.

The fact that the upper bound of order $1 / \bar{F}(x)$ is attainable follows from the following example.

Example 7.2. Let $F$ be a discrete subexponential distribution with $f_{0}>0, f_{1}, f_{2}, \ldots$ being point probabilities. A discrete-time regenerative process $Z$ is constructed as follows. At the start of a cycle, an RV $X$ with distribution $F$ is drawn. If $X=0$, we take $R=1$ and $\xi=-b$. 
If $X=x>0$, we take $R=\varphi(x)$ for some suitable $\varphi(x) \uparrow \infty, Z_{0}=\cdots Z_{\varphi(x)-2}=0$, and $\xi=Z_{\varphi(x)-1}=x$ (we then need to choose $b$ such that $\mathbb{E} \xi<0$ ).

The question is whether all rates are attainable. To discuss this point, let $\varphi(x) \uparrow \infty$. By Theorem 7.1, $\varphi$ must satisfy $\varphi(x)=\mathrm{O}(1 / \bar{F}(x))$. Conversely, the construction works if (7.3) holds and gives a risk process such that $\tau$ grows at rate at least $\varphi(x)$.

For example, if $F$ is regularly varying with index $\alpha>1$, this allows for growth rates $\varphi(x)$ of order $x^{\beta}$ with $1<\beta<\alpha$, whereas $e(x)$ is only of order $x$.

\section{References}

[1] Albrecher, H., Asmussen, S. and Kortschak, D. (2012). Tail asymptotics for dependent subexponential differences. Siberian Math. J. 53, 965-983.

[2] Asmussen, S. (2003). Applied Probability and Queues, 2nd edn. Springer, New York.

[3] Asmussen, S. and Albrecher, H. (2010). Ruin Probabilities, 2nd edn. World Scientific, Hackensack, NJ.

[4] Asmussen, S. AND Biard, R. (2011). Ruin probabilities for a regenerative Poisson gap generated risk process. Europ. Actuarial J. 1, 3-22.

[5] Asmussen, S. And KlüPPElberg, C. (1996). Large deviations results for subexponential tails, with applications to insurance risk. Stoch. Process. Appl. 64, 103-125.

[6] Asmussen, S. And KortschaK, D. (2011). Second order approximations for normalized ruin times in the presence of heavy tails. Stoch. Systems 3, 591-633.

[7] Asmussen, S., Klüppelberg, C. and Sigman, K. (1999). Sampling at subexponential times, with queueing applications. Stoch. Process. Appl. 79, 265-286.

[8] Asmussen, S., Schmidli, H. And Schmidt, V. (1999). Tail probabilities for non-standard risk and queueing processes with subexponential jumps. Adv. Appl. Prob. 31, 422-447.

[9] Baccelli, F. And Foss, S. (2004). Moments and tails in monotone-separable stochastic networks. Ann. Appl. Prob. 14, 612-650.

[10] Balkema, A. A. And de Haan, L. (1974). Residual life time at great age. Ann. Prob. 2, $792-804$.

[11] Balkema, A. A., Resnick, S. I. And Klüppelberg, C. (1993). Densities with Gaussian tails. Proc. London Math. Soc. 66, 568-588.

[12] Bingham, N. H., Goldie, C. M. and Teugels, J. L. (1987). Regular Variation. Cambridge University Press.

[13] Buörk, T. ANd Grandell, J. (1988). Exponential inequalities for ruin probabilities in the Cox case. Scand. Actuarial J. 1988, 77-111.

[14] Breiman, L. (1965). On some limit theorems similar to the arc-sine law. Theory Prob. Appl. 10, 323-331.

[15] Cline, D. B. H. and Samorodnitsky, G. (1994). Subexponentiality of the product of independent random variables. Stoch. Process. Appl. 49, 75-98.

[16] Denisov, D. AND Zwart, B. (2007). On a theorem of Breiman and a class of random difference equations. J. Appl. Prob. 44, 1031-1046.

[17] Denisov, D., Foss, S. And Korshunov, D. (2010). Asymptotics of randomly stopped sums in the presence of heavy tails. Bernoulli 16, 971-994.

[18] Embrechts, P., Klüppelberg, C. And Mikosch, T. (1997). Modelling Extremal Events. For Insurance and Finance. Springer, Berlin.

[19] Foss, S. AND ZACHARY, S. (2002). Asymptotics for the maximum of a modulated random walk with heavytailed increments. In Analytic Methods in Applied Probability (Amer. Math. Soc. Transl. Ser. 2 207), American Mathematical Society, Providence, RI, pp. 37-52.

[20] Foss, S., Konstantopoulos, T. and Zachary, S. (2007). Discrete and continuous time modulated random walks with heavy-tailed increments. J. Theoret. Prob. 20, 581-612.

[21] Foss, S., Korshunov, D. And Zachary, S. (2011). An Introduction to Heavy-Tailed and Subexponential Distributions. Springer, New York.

[22] Goldie, C. M. And Resnick, S. (1988). Distributions that are both subexponential and in the domain of attraction of an extreme-value distribution. Adv. Appl. Prob. 20, 706-718.

[23] KlüPPelberg, C., KyPrianou, A. And Maller, R. (2004). Ruin probabilities and overshoots for general Lévy insurance risk processes. Ann. Appl. Prob. 14, 1766-1801. 